\title{
Perspectives on critical care nursing in China
}

Chun-yan Li, RN; BScN, Nursing Director, Chao Yang Hospital, Beijing, China, David Chan, RN; BScN, MN (Acute Care), Nurse Specialist (ICU), Prince of Wales Hospital, Hong Kong.

E-mail: milama@163.com or davidchan123@hotmail.com

Key words: China critical care history intensive care nursing

\section{SUMMARY}

- $\quad$ This paper summarises the historical development of intensive care services in China over the last 25 years.

- $\quad$ The history of intensive care in China began in 1982 when Peking Union Medical College developed the first intensive care unit in its hospital in Beijing.

- $\quad$ The rapid developmental phase of critical care in China started in 1991 when the Ministry of Health claimed the statement, "Developing the intensive care specialty in our country".

- $\quad$ With the opening door policy of the Chinese Government and the rapid development of economic expansion, advanced medical technology and new nursing concepts were brought into China. Four major cities in China now have intensive care units with good facilities and standards, namely: Beijing, Shanghai, Guangzhou, and Chengdu, and many other large hospitals in China now have well-equipped intensive care units.

- What China now needs is a system to standardise intensive care education and standards throughout the country.

\section{INTRODUCTION}

The development of critical care in China started during the 196070s. With the rapid development of medical technology and an increase in the number of critically ill patients, many hospitals throughout the country started developing recovery rooms in operating theatres for providing close observation and prompt intervention to patients who had received major surgery (Liu \& Chen, 2006, p20). The impetus for setting up recovery rooms was due to the rapid development of medical technology, especially the respiratory and cardiovascular monitoring equipment. This change was also influenced by the severity of patients' health problems, especially those who suffered from multiple organ failure. These problems could not be easily dealt with by doctors and nurses in general wards. It was therefore necessary to centralise all critically ill patients in one area to receive specialised treatment and care (Liu \& Chen, 2006, p20). Such specialised care required staff who were trained appropriately to work in these designated areas.

\section{THE DEVELOPMENT OF INTENSIVE CARE}

Towards the end of the 1970s, the open door policy of the Chinese Government (Liu \& Chen, 2006, p20) allowed the first batch of medical and nursing scholars to go overseas to pursue advanced training in critical care medicine and critical care nursing in countries such as the United States, United Kingdom and Australia. This was an instrumental factor in the development of the intensive care specialty in China, which occurred in three phases: foundational, developmental, and maturational.

\section{Foundational phase}

The foundational phase began in 1982 when the first intensive care unit (ICU) was established at the Peking Union Medical College (PUMC) Hospital in Beijing. During the same period, other hospitals in China also started to establish ICUs, for example the Central Hospital in Tientsin. The first ICUs in China were small, with only a few beds, because of limited resources and trained staff. Nowadays, some ICUs, such as the one in the An Zhen Hospital in Beijing, as many as 40 beds which are well-resourced with modern equipment. Along with the development of ICUs in China, monitoring technology was also rapidly expanding to a level of sophistication comparable with that of the western world. The implementation of new technologies in ICU has helped clinicians to make better diagnoses and more efficient clinical decision-making, which in turn has helped to reduce patient mortality (Liu \& Chen, 2006, p21).

\section{Developmental phase}

The developmental phase began in 1991, when the Ministry of Health announced that the development of the intensive care specialty in China was a priority (Liu \& Chen, 2006, p21). As a result, many large and medium size hospitals established their ICUs, and started to formalise their standards of practice. Most big cities now have excellent ICU facilities and standards. Examples of these hospitals are in Beijing (PUMC Hospital, Fu Wai Hospital, Beijing University Number 1 Hospital, Beijing University Number 3 Hospital, An Zhen Hospital, Chao Yang Hospital, Friendship Hospital), Shanghai (Xin Hua Hospital, Children's Hospital, Ren Ji Hospital), Guangzhou (Guangdong Cardiovascular Hospital, Guangdong Province People's Hospital), and Chengdu (Hua Xi Hospital). Most of these hospitals have multi-disciplinary ICUs and some are specialty-based ICUs. Many of the ICUs are managed by the Accident and Emergency Department Director. 


\section{* Perspectives on critical care nursing in China *}

With the establishment of ICU in China, the concept of patient care also changed. It moved away from the traditional medical model of treating organs towards a consideration of the whole person, in terms of their healthcare and social needs. This signalled a departure from fragmented and task-oriented care to the use of a holistic approach to manage the patient's problems. For example, in the past adult respiratory distress syndrome (ARDS) was regarded as a lung problem only, but now it is understood as a syndrome that is associated with major surgery, trauma, infection or other health problems. Previously, ARDS was managed by using antibiotics and mechanical ventilation, but nowadays, it is managed by focusing on diagnosis, providing adequate supportive treatment, and instituting different types of lung protective ventilation strategies such as prone ventilation, inhaled nitric oxide, extra-corporeal membrane oxygenation (ECMO) and protocol weaning. With regard to nursing, ICU nurses have also shifted the focus of care of such patients from provision of primarily physical care (for example, ventilatory care) to provision of care in a holistic manner, including physiological care (for example, ensuring adequate oxygenation, neurological function, fluid and electrolytes, nutrition) and psycho-social care (for example, early detection and management of patient's anxiety and other psychological responses due to problems in psychological, social and emotional aspects). The way that critically ill patients' health problems were managed was also influenced by visits from specialist consultants from other countries to Chinese ICUs.

\section{Maturational phase}

After the year 2000, the development of ICU in China entered the maturational phase. With the rapid economic expansion in China, many provinces have built large, modern hospitals with well equipped ICUs. The major health care authorities in China (for example, the Health Ministry, the Chinese Medical Association, and the Chinese Nursing Association) are now helping these hospitals to form standards and to develop formal education programmes related to intensive care specialties. Although most nurses in modern China are still trained in hospitals and health care schools, university-based education is increasing, with support from the various medical schools.

Specialty training programmes in intensive care are currently organised by professional associations (for example, the Chinese
Nursing Association) and by individual hospitals. The Ministry of Health is also planning to standardise specialty training throughout the country in the near future (Liu \& Chen, 2006, p. 21). China has also started to consider specialisation in intensive care, such as the development of Nurse Specialist positions (Liu \& Chen, 2006, p. 21).

In 2003, the ICU committees of the Chinese Nursing Association and the Chinese Medical Association were set up. The establishment of these professional organisations signified a very important milestone in the development of critical care in China, because they helped the development of ICU services by standardising ICU standards, improving ICU education, and fostering new and advanced practices among ICUs throughout the country.

\section{CHALLENGES}

China is an enormous country with a huge population of about 13 billion people but the nursing workforce in health care is largely inadequate, with only 1.3 million nurses compared to 2 million doctors. By ratio, this works out at one doctor to 650 people (similar to Hong Kong), compared to only one nurse to 1000 people (one nurse to 300 people in Hong Kong). It is estimated that the nurse-bed ratio in ICU is between 1:1 and 1:3 (Liu \& Chen, 2006, p. 21). Therefore, there is a need to recruit more nurses into nursing and also to encourage nurses to specialise in critical care. In this respect, there is a need to develop active campaign strategies and to promote nurses' work, as well as raising the image of nurses in China. Hopefully, with support and through collaboration with other critical care nurses worldwide, nurses in China can overcome some of these problems.

In August 2006, the 14th Congress of the Asia-Pacific Association of Critical Care Medicine (APACCM) conference will be held in Beijing (www.apaccm.org.cn). This will be a great opportunity for ICU doctors and nurses in China to widen their perspectives in the intensive care arena, and to help foster the development of intensive care medicine and nursing in China.

\section{Reference}

Liu SJ \& Chen YQ (2006). Standard Training Course for Critical Care Nursing. Beijing: People's Military Medical Press, pp 2021. 